\title{
piRNA pathway is essential for generating functional oocytes in golden hamster
}

Hongdao Zhang ${ }^{1, \#}$, Fengjuan Zhang ${ }^{1, \#}$, Jinghua Chen ${ }^{2, \#, ~ M i n g z h i ~ L i ², \#, ~ X i a o l o n g ~ L v ' ~}{ }^{1}$, Yali Xiao ${ }^{1}$,

Zhaozhen Zhang ${ }^{2}$, Li Hou ${ }^{1}$, Yana Lai², Wen Xiao ${ }^{1}$, Aihua Zhang ${ }^{2}$, Heling Fu², Jianli Zhou ${ }^{2}$,

Feiyang Diao ${ }^{2,3}$, Aimin $\mathrm{Shi}^{2}$, Youqiang $\mathrm{Su}^{2}$, Wentao Zeng ${ }^{2, *}$, Ligang $\mathrm{Wu}^{1, *}$, Jianmin $\mathrm{Li}^{2, *}$

1 State Key Laboratory of Molecular Biology, Shanghai Key Laboratory of Molecular Andrology, Center for Excellence in Molecular Cell Science, Shanghai Institute of Biochemistry and Cell Biology, Chinese Academy of Sciences, University of Chinese Academy of Sciences, Shanghai 200031, China

2 Model Animal Research Center of State Key Laboratory of Reproductive Medicine, Jiangsu Laboratory Animal Center, Jiangsu Animal Experimental Center of Medicine and Pharmacy, Jiangsu Animal of Model, Department of Cell Biology, Animal Core facility, Key Laboratory of Model Animal, Collaborative Innovation Center for Cardiovascular Disease Translational Medicine, Nanjing Medical University, Nanjing 211166, China

3 Clinical Center of Reproductive Medicine, the First Hospital Affiliated with Nanjing Medical University, Nanjing, China

\# These authors contributed equally to this work.

* Correspondence:

Jianmin Li, jianminlilab@njmu.edu.cn

LigangWu, lgwu@sibcb.ac.cn

Wentao Zeng, wentaozeng@njmu.edu.cn 


\begin{abstract}
Piwi-interacting RNAs (piRNAs) are small RNAs predominantly expressed in germ cells that are critical for gametogenesis in various species. However, PIWI-deficient female mice are fertile and mouse oocytes express a panel of small RNAs that do not appear widely representative of mammals, and piRNA function in the oogenesis of other mammals has therefore remained elusive. Recent studies revealed the small RNA and PIWI transcriptional profiles in golden hamster oocytes more closely resemble that of humans than mice. Herein, we generated PIWIL1-, PLD6- and MOV10L1- deficient golden hamsters and found that all female mutants were sterile, with embryos arrested at the two-cell stage. In PIWIL 1 mutant oocytes, we observed transposon accumulation and broad transcriptomic dysregulation, while zygotic gene activation was impaired in early embryos. Intriguingly, PIWIL1-piRNAs exhibited a unique, preferential silencing of endogenous retroviruses (ERVs), whereas silencing LINE1s depended on both PIWIL1- and PIWIL3-piRNAs. Moreover, we showed that piRNAs participate in the degradation of maternal mRNAs in MII oocytes and embryos via partially complementary targets. Together, our findings demonstrate that piRNAs are indispensable for generating functional oocytes in golden hamster, and show the informative value of this model for functional and mechanistic investigations of piRNAs, especially those related to female infertility.
\end{abstract}




\section{Introduction}

Over the past two decades, substantial progress has been made towards understanding the transcriptional and post-transcriptional regulatory systems in gene expression mediated by small RNAs. Among these, PIWI-interacting RNAs (piRNAs) are single-stranded small RNAs, 18-30 nt in length, that are predominantly expressed in germ cells and bind with PIWI proteins to form piRNA-induced silencing complexes (piRISCs), which provide diverse and indispensable functions in gametogenesis (1-6). The primary function of the piRNA pathway is to suppress active transposable elements (TEs) by post-transcriptional gene silencing (PTGS) through piRISCmediated slicer activity, as well as transcriptional gene silencing (TGS) through guiding DNA methylation or histone modification $(7,8)$. Moreover, increasing evidence has shown that the piRNA pathway could also regulate mRNAs and lncRNAs (9-12), and are functional in tissue regeneration $(13,14)$, tumor biology $(15,16)$, and embryogenesis (17-19).

In Drosophila and Zebrafish, disruption of any of the PIWI paralogs severely impairs the fertility of both males and females (20-23). In mammals, the piRNA pathway has been primarily studied in mice, whose genome encodes three PIWIs, including PIWIL1 (MIWI), PIWIL2 (MILI), and PIWIL4 (MIWI2). Disruption of any PIWIs in mice leads to spermatogenesis arrest and sterility in males (24-26). However, female mice remain fertile in the absence of PIWIs, as well as other genes that are critical for piRNA biogenesis (24-30). Unlike many other mammals, endo-siRNAs are the most abundant small RNAs in mouse oocytes, which might be attributable to the expression of a unique isoform of Dicer $\left(\right.$ Dicer $\left.^{\mathrm{O}}\right)$ that is highly effective in producing endo-siRNAs, while the abundance of piRNAs is dwarfed in contrast (31-33). These differences suggest that the dispensability of the piRNA pathway in female fertility previously reported in mice may not be representative of all mammals, including humans, and that the role of piRNAs warrants closer examination in other animals. Golden hamster (Mesocricetus auratus) belongs to the Cricetidae family of rodents and has been used as a model in studying development and reproductive biology for several decades (34). Moreover, the golden hamster genome encodes all four PIWIs, with an oocyte small RNA transcriptional profile that more closely resembles the human profile than the mouse (Fig. 1A-B) (1, 35). In particular, piRNAs and PIWIs are highly expressed, thus providing an informative animal model to investigate the potential involvement of the piRNA pathway in 
female gametogenesis. In this study, we demonstrate the essential function of the piRNA pathway in female reproduction in golden hamster. Furthermore, we provide evidence that PIWIL1 and piRNAs are functional in mediating transposon silencing and degradation of maternal mRNAs, and that they play an essential role in oogenesis and the initiation of ZGA to sustain the development of early embryos.

\section{Results}

Although CRISPR/Cas9 technology has been used successfully in golden hamsters $(36,37)$, the production of genetically-modified golden hamsters has remained a challenge (36). We optimized several critical steps for producing mutant golden hamsters. First, we injected CRISPR/Cas9 into 2-cell embryos, rather than the pronucleus, and then cultured the injected embryos in HEMC9+PVA medium, which significantly increased the survival rate of embryos by more than $50 \%(36,38,39)$ (data not shown). Furthermore, we generated an albino (Tyr-deficient) golden hamster strain to simplify the procedure for identifying genome-edited pups by their coat color, while true pregnant females were used as recipients (Fig. s1).

We generated PIWIL1-deficient golden hamster strains by two-cell embryo injection (Fig. 1C) with CRISPR/Cas9 using two sgRNAs targeting exon 6 and exon 17 of PIWIL1, respectively. Two PIWIL 1 mutant strains were obtained (Fig. 1D and Fig. s2A) and both mutations caused frame shifts that resulted in premature stop codons in PIWIL1 mRNAs (Fig. s2B). In homozygous PIWIL1deficient animals, no PIWIL1 protein was detectable in the ovaries, MII oocytes, early embryos, or testes by western blotting (Fig. 1E) and immunostaining (Fig. 1F-G and Fig. s2C-D), thus confirming the complete loss of PIWIL1 expression. Both male and female PIWIL1 homozygous mutants appeared physiologically normal but were infertile (Fig. 2A). The two independent PIWILI mutants (PIWIL $1^{\mathrm{m} 1 / \mathrm{m} 1}$ and $P I W I L 1^{\mathrm{m} 2 / \mathrm{m} 2}$ ) showed identical results, which ruled out potential offtarget effects of Cas9/sgRNA.

The testes of PIWIL1 homozygous mutants were substantially smaller than their WT or heterozygous counterparts (Fig. s3A-B). No mature sperm was found in the caput and cauda epididymis of mutants (Fig. s3D-E). Histological examination revealed that round and elongated spermatids were completely absent and that spermatogenesis was uniformly arrested at the 
pachytene stage (Fig. s3C), which was further confirmed by immunostaining of $\gamma \mathrm{H} 2 \mathrm{AX}$ (Fig. s3F) (24). These observations indicated that PIWIL1 is essential for spermatogenesis in golden hamsters and that these mutants showed comparable developmental abnormality to PIWIL1 mutant male mice, as reported previously (26).

PIWIL1 mutant females displayed normal ovarian histology (Fig. s4A), with no obvious differences from the WT in the number of ovulated metaphase II (MII) oocytes (Fig. s4B) or morphology of the MII spindle (Fig. s4C). We found that MII oocytes from PIWIL1 $1^{\mathrm{m} 1 / \mathrm{m} 1}$ could be successfully fertilized in vivo and subsequently form normal pronuclei at 9 hours post egg activation (9 PEA) (Fig. 2C and Fig. s5A). However, in contrast to the WT zygotes, PIWIL $1^{\mathrm{m} 1 / \mathrm{ml}}$ zygotes were arrested at the two-cell stage in vivo (Fig. 2C-D and Fig. s5A). Those arrested two-cell embryos could be maintained up to 52-54 PEA, but beyond that time these embryos appeared to die and degrade in the oviduct. To confirm the embryogenesis defects, zygotes (collected at 9 PEA) were isolated from the oviduct and cultured in vitro. A similar two-cell arrest phenotype was observed (Fig. 2D and Fig. s5B) and the embryos broke down after 52 PEA, while WT embryos grew continuously beyond the four-cell stage. Interestingly, the microtubule bridge between two sister cells was absent in PIWIL1 mutant embryos at 33 to 52 PEA (Fig. s5D), indicating abnormal cell division (40). These observations were confirmed in an independent PIWIL1 mutant stain (PIWIL $1^{\mathrm{m} 2 / \mathrm{m} 2}$ ) (Fig. $\mathrm{s} 5 \mathrm{C}$ ), suggesting the embryogenesis deficient phenotype is attributable to PIWIL1.

To rule out the possibility that PIWILI disruption caused defects in the oviduct and ovary that could lead to sterility, we transferred two-cell embryos at 33 PEA from PIWIL $1^{\mathrm{m} 1 / \mathrm{ml} 1}$ females mated with WT males into WT recipient females (Fig. s6A). The embryos failed to pass the two-cell stage in these surrogate females (Fig. 2B). Conversely, embryos from WT albino hamsters were transferred into the oviducts of PIWIL $1^{\mathrm{m} 1 / \mathrm{m} 1}$ females (Fig. $\mathrm{s} 6 \mathrm{~B}$ ), and the PIWIL $1^{\mathrm{m} 1 / \mathrm{m} 1}$ surrogate females gave birth to albino hamsters with normal efficiency (Fig. 2E). In addition, we found that PIWIL 1 heterozygotes appeared physiologically normal and gave birth to $P I W I L 1^{\mathrm{m} 1 / \mathrm{m} 1}$ offspring at the expected ratio. These results suggested that the loss of maternal PIWIL1 might be the cause of oocyte incompetence and contributed to early embryogenesis arrest.

To further investigate whether a similar phenotype could also be observed upon knockout of 
other essential genes in the piRNA pathway, we generated mutants of PLD6 (or ZUCCHINI or MITOPLD) endonuclease and MOV1OL1 helicase, respectively, which are essential for piRNA biogenesis (27-30) (Fig. s7A-B, Fig. s8A-B). The resulting heterozygous PLD6 and MOV10L1 mutant males and females showed normal reproductive ability, whereas homozygous PLD6- and MOV10L1-deficient male and female hamsters were sterile (Fig. s7C-D, Fig. s8C-D). Notably, PLD6 and MOV1OL1 mutants displayed embryonic arrest at the two-cell stage similarly to the PIWIL1 mutant (Fig. s7E-F, Fig. s8E-F), thus confirming the indispensable role of the piRNA pathway in female fertility in golden hamster.

Because knockout of PIWIL1, PLD6, and MOV1OL1 all resulted in similar phenotypes, we then focused on the PIWIL1 mutants to investigate the underlying mechanisms that lead to abnormalities in oocytes. The oocytes and embryos at different developmental stages were collected from WT and $P I W I L 1^{\mathrm{m} 1 / \mathrm{m} 1}$ females for single-cell small RNA and transcriptome sequencing with exogenous spike-in. The sequencing results revealed the presence of three populations of differently sized piRNAs, including 18-21 nt (19 nt-piRNA), 22-24 nt (23 nt-piRNA), and 28-30 nt (29 ntpiRNA), the relative abundances of which were dynamically regulated during oogenesis and early embryogenesis (Fig. 3A). We characterized these piRNAs and found all of them showed a strong preference for uridine $(\mathrm{U})$ at their 5' end and other typical piRNA characteristics (Fig. s9-10)(7, 8). Upon $\mathrm{NaIO}_{4}$ treatment, 23 nt-piRNAs and 29 nt-piRNAs remained intact while 19 nt-piRNAs almost completely disappeared (Fig. s10A). These findings indicated that $23-$ and 29 nt-piRNAs possessed 2'-O-methylation at their 3' end, while the 19 nt-piRNAs did not, which was consistent with the features of long piRNAs and os-piRNAs previously reported in human and monkey oocytes (1). In PIWIL1 mutants, 23 nt-piRNAs and 29 nt-piRNAs were completely undetectable, while 19 nt-piRNAs and miRNAs were unaffected (Fig. 3A and Fig. s10B). We further sequenced small RNAs associated with PIWIL1 and found that 23- and 29 nt-piRNA both co-purified with PIWIL1 in WT MII oocytes but were absent in PIWIL1-deficient oocytes (Fig. s10C-D), consistent with observations of diminished 23- and 29 nt-piRNAs in the PIWIL1 mutants (Fig. 3A). Furthermore, the 23- and 29 nt-piRNA shared the same piRNA clusters and 5' ends (Fig. s11), which supported the likelihood that both 23 nt-piRNAs and 29-nt piRNAs are associated with PIWIL1 in hamster oocytes (35). In the testes, only the 29 nt-piRNAs were found to bind with PIWIL1 and these 
piRNAs were completely absent in PIWIL1 mutants (Fig. s12), which suggested that PWIL1associated piRNAs in hamster testes shared similar characteristics and function to their counterparts in mouse testes $(4,5)$.

Approximately $35 \%$ of the piRNAs could be mapped to known regions of the genome. Among them, most were mapped to TEs (Fig. s13 and Fig. s14), which was consistent with the conserved function of piRNAs in silencing TEs $(7,8)$. Our transcriptome data from oocytes and embryos at different developmental stages revealed that TEs accumulated during follicle development and started to decrease after the germinal vesicle (GV) stage in WT (Fig. s15A), possibly due to suppression by the piRNA pathway. Intriguingly, a minor wave of TE activation was observed in one-cell and two-cell embryos, reminiscent of similar observations reported during early embryogenesis of other mammals $(41,42)$. In the PIWIL1 mutant, TE expression was significantly up-regulated, especially from the GV oocyte to one-cell embryo stages (Fig. 3B). This trend was sustained until 33 PEA, at which point PIWIL1-mutant embryos underwent developmental arrest and subsequent degradation. In particular, ERVs were the most highly up-regulated TEs, followed by LINE1s (Fig.s15B). The ERV-related PIWIL1-piRNAs showed an obvious bias to the antisense of ERV mRNAs (Fig.3C and Fig. s16) and a strong Ping-Pong signature (Fig. 3D and Fig. s17), which suggested that involvement of PIWILI cleavage guided by antisense piRNAs in silencing ERVs. Notably, the ERV-related PIWIL3-piRNAs were almost completely depleted in oocytes and embryos upon PIWIL1 disruption (Fig. 3C, Fig. s16B and Fig. s17), implying that generation of ERV-related PIWIL3-piRNAs, to some extent, depends on PIWIL1, possibly through inter-PingPong crosstalk between two different PIWIL proteins $(43,44)$. These results highlight the nonredundant and essential role of PIWIL1 in silencing ERVs.

For LINE1s, both PIWIL1- and PIWIL3-piRNAs showed an obvious Ping-Pong signature (Fig. 3D). Intriguingly, the abundance of LINE1-related PIWIL3-piRNAs, as well the Ping-Pong signature, were increased following PIWIL1 knockout (Fig. 3C-D, Fig. s16), which was potentially caused by high accumulation of TE mRNAs and/or primary piRNA transcripts in the PIWIL1 mutants. These results suggested that both PIWIL1 and PIWIL3 were involved in LINE1 silencing and exhibited redundant functions. The differential activity in silencing ERVs and LINE1s by PIWIL1 and PIWIL3 might be attributable to the preferential interaction of individual PIWI proteins 
with the pre-piRNA processing complexes, which warrants further investigations.

In light of these findings, we speculated that the accumulation of TEs might not be the exclusive reason for the arrest of two-cell stage embryos in PIWIL1 mutants. Principal component analysis (PCA) for gene expression between stages showed that the oocytes and 1-cell embryos of mutant and WT grouped tightly and were distinguishable from other stages, whereas PIWIL1mutant embryos after 33 PEA clustered separately from WT (Fig. 4A and Fig. s18). Developmental trajectory assays showed that PIWIL1-mutant embryos branched off toward a different cell fate from that of WT embryos after 33 PEA (Fig. 4B), which became developmentally incompetent and died within 1-2 days in vivo and in vitro. Quantification of mRNAs showed 254 and 1186 differentially expressed genes (DEGs) in 1-cell and 2-cell embryos of PIWIL1-mutants, respectively. Notably, the DEGs significantly elevated in WT embryos were rarely up-regulated in PIWIL1 mutant embryos (Fig. 4C and Fig. s19), indicating that minor and major zygote gene activation (ZGA) was severely inhibited in PIWIL1-mutants.

Initiation of ZGA is known to coincide with the degradation of maternal mRNAs, which is started in GV oocytes to remove repressive factors and to enable zygotic transcription, as well as to permit the establishment of embryonic patterning (45), raising the possibility that the inhibition of ZGA in PIWIL1-deficient embryos might be trigged by impaired decay of maternally deposited transcripts, with deleterious consequences. To examine this hypothesis, we investigated the relative mRNA abundance in each stage and found that the decay rate of many rapidly degraded mRNAs dramatically decreased in PIWIL1 mutants during the MII oocyte to 1-cell transition (Fig. 4D).

Previous studies showed that piRNAs affect the expression of mRNAs by two distinct mechanisms: one relies on PIWIL1 slicer activity to cleave almost perfectly complementary targets; the other mechanism is to trigger deadenylation of partially complementary targets via recruitment of the deadenylation complex $(9,10,19,46)$. We observed a significant enrichment of target sequences that were partially complementary to PIWIL1-piRNAs on those mRNAs with impaired degradation. The number of targets correlated with the changes in the degradation rate of mRNAs upon PIWIL1 deletion (Fig. 4E), as did the abundance of piRNAs (Fig. s20A), which suggested the involvement of PIWIL1 and its associated piRNAs in controlling maternal mRNA stability. Notably, the PIWIL1 deficiency in MII oocytes also resulted in impaired degradation of many predicted target 
mRNAs of PIWIL3-piRNAs whose abundance was affected in the absence of PIWIL1 (Fig. s14, Fig. s20B-C). These results implied that PIWIL1 might also indirectly regulate additional maternal mRNA degradation through PIWIL3 and its associated piRNAs.

\section{Discussion}

Mice are the most commonly used animal model for studying gene function and human disease. However, growing evidence shows that mice may not be as widely representative of mammals in some genetic and physiological aspects as previously thought (34). piRNAs appear to be one such case. Previous studies have shown that PIWIs and piRNAs are essential for female fertility in flies and fish (20-23), but dispensable for female fertility in mice (24-30), a discrepancy likely due to the lack of PIWIL3 in the mouse genome, as well as mouse-specific expression of endo-siRNAs that functionally overlap with piRNAs for silencing TEs in oocytes $(31,47)$. In this study, we showed that the piRNA pathway is essential for female fertility in golden hamsters. In addition to their highly conserved function in silencing TEs, we show that piRNAs also function in the regulation of maternal mRNA stability in oocytes and embryos, and that disruption of the piRNA pathway leads to gene expression dysregulation in oocytes and failure to initiate ZGA in embryogenesis. Previous studies have shown that in Drosophila, Aubergine (PIWI homolog) and its associated piRNAs recruit Smaug and the CCR4 deadenylation complexes to eliminate specific maternal mRNAs in the early embryo (19); and in Aedes aegypti, satellite repeat-derived piRNAs degrade maternally deposited transcripts in the zygote (17), indicating that the role of piRNAs in maternal mRNA clearance is conserved across vertebrate and invertebrate species. During the preparation of this report, two other groups have described similar observations (albeit in non-peer-reviewed preprints) corroborating the indispensable functions of PIWIL1 and MOV1OL1 in female fertility in golden hamster $(48,49)$. Given the small size, ease of maintenance, high reproductive performance, and most importantly, similar patterns of PIWIs and piRNAs expression in oocytes compared to humans, we propose an expanded role for golden hamster as an instructive and tractable representative model for mammalian reproduction. In particular, the investigation of piRNA-related functions in females can yield valuable insights with potential clinical implications for fertility-related disorders. 


\section{Acknowledgment}

We thank all members of Ligang Wu's and Jianmin Li's laboratory for their discussion and comments on this project. We thank Zhixue Li for bioinformatics analyses, Yuanyuan Song for library construction. We thank Yaochen $\mathrm{Xu}$ for his assistance with high-performance computing. We are grateful to the HPC storage and network service platform of SIBCB for supplying the computing resources. We thank Dr. Haruhiko Siomi (Keio University School of Medicine, Tokyo, Japan) for sharing unpublished data. We are also grateful to Ying Huang, Mofang Liu, and Yang Yu for their critical comments on the manuscript.

\section{Funding}

This work was supported by the following funding: the Strategic Priority Research Program of the Chinese Academy of Sciences (XDB19040102), the National Key R\&D Program of China (2017YFA0504401), and the National Natural Science Foundation of China (31970607 and 31470781) to Ligang Wu; the National Basic Research (973) Program of China (2009CB941700) and the National Natural Science Foundation of China (31171443) to Jianmin Li; the National Key Research and Development Program of China (2018YFC1003800), the National Natural Science Foundation of China (31871507, 31471351, and 31271538), the National Basic Research (973) Program of China (2014CB943200 and 2013CB945500), and the National Natural Science Foundation of Jiangsu Province (BK20140061) to You-qiang Su.

\section{Competing interests}

The authors declare that they have no competing interests. 


\section{Reference}

1. Q. Yang et al., Single-cell CAS-seq reveals a class of short PIWI-interacting RNAs in human oocytes. Nature communications 10, 3389 (2019).

2. V. V. Vagin et al., A distinct small RNA pathway silences selfish genetic elements in the germline. Science 313, 320-324 (2006).

3. $\quad$ N. C. Lau et al., Characterization of the piRNA complex from rat testes. Science 313, 363367 (2006).

4. S. T. Grivna, E. Beyret, Z. Wang, H. F. Lin, A novel class of small RNAs in mouse spermatogenic cells. Gene Dev 20, 1709-1714 (2006).

5. A. Girard, R. Sachidanandam, G. J. Hannon, M. A. Carmell, A germline-specific class of small RNAs binds mammalian Piwi proteins. Nature 442, 199-202 (2006).

6. A. Aravin et al., A novel class of small RNAs bind to MILI protein in mouse testes. Nature 442, 203-207 (2006).

7. D. M. Ozata, I. Gainetdinov, A. Zoch, D. O'Carroll, P. D. Zamore, PIWI-interacting RNAs: small RNAs with big functions. Nature Reviews Genetics 20, 89-108 (2019).

8. B. Czech et al., piRNA-Guided Genome Defense: From Biogenesis to Silencing. Annual Review Of Genetics, Vol 52 52, 131-157 (2018).

9. P. Zhang et al., MIWI and piRNA-mediated cleavage of messenger RNAs in mouse testes. Cell research 25, 193-207 (2015).

10. T. Watanabe, E. C. Cheng, M. Zhong, H. F. Lin, Retrotransposons and pseudogenes regulate mRNAs and IncRNAs via the piRNA pathway in the germline. Genome research $\mathbf{2 5}, 368$ 380 (2015).

11. L.-T. Gou et al., Pachytene piRNAs instruct massive mRNA elimination during late spermiogenesis. Cell research 24, 680-700 (2014).

12. Y. A. Sytnikova, R. Rahman, G. W. Chirn, J. P. Clark, N. C. Lau, Transposable element dynamics and PIWI regulation impacts IncRNA and gene expression diversity in Drosophila ovarian cell cultures. Genome research 24, 1977-1990 (2014).

13. P. W. Reddien, N. J. Oviedo, J. R. Jennings, J. C. Jenkin, A. S. Alvarado, SMEDWI-2 is a PIWI-like protein that regulates planarian stem cells. Science 310, 1327-1330 (2005).

14. F. Rizzo et al., Timed Regulation of P-Element-Induced Wimpy Testis-Interacting RNA Expression During Rat Liver Regeneration. Hepatology 60, 798-806 (2014).

15. S. Shi, Z. Z. Yang, S. H. Liu, F. Yang, H. F. Lin, PIWIL1 promotes gastric cancer via a piRNA-independent mechanism. Proceedings of the National Academy of Sciences of the United States of America 117, 22390-22401 (2020).

16. F. Li et al., piRNA-independent function of PIWIL1 as a co-activator for anaphase promoting complex/cyclosome to drive pancreatic cancer metastasis (vol 22, pg 425, 2020). Nature cell biology 22, 751-751 (2020).

17. R. Halbach et al., A satellite repeat-derived piRNA controls embryonic development of Aedes. Nature 580, 274-+ (2020).

18. S. R. Mani, H. Megosh, H. F. Lin, PIWI proteins are essential for early Drosophila embryogenesis. Developmental biology 385, 340-349 (2014).

19. C. Rouget et al., Maternal mRNA deadenylation and decay by the piRNA pathway in the early Drosophila embryo. Nature 467, 1128-U1144 (2010).

20. C. J. Li et al., Collapse of Germline piRNAs in the Absence of Argonaute3 Reveals Somatic 
piRNAs in Flies. Cell 137, 509-521 (2009).

21. S. Houwing, E. Berezikov, R. F. Ketting, Zili is required for germ cell differentiation and meiosis in zebrafish. Embo Journal 27, 2702-2711 (2008).

22. S. Houwing et al., A role for Piwi and piRNAs in germ cell maintenance and transposon silencing in zebrafish. Cell 129, 69-82 (2007).

23. D. N. Cox, A. Chao, H. F. Lin, piwi encodes a nucleoplasmic factor whose activity modulates the number and division rate of germline stem cells. Development 127, 503-514 (2000).

24. M. A. Carmell et al., MIWI2 is essential for spermatogenesis and repression of transposons in the mouse male germline. Developmental cell 12, 503-514 (2007).

25. S. Kuramochi-Miyagawa et al., Mili, a mammalian member of piwi family gene, is essential for spermatogenesis. Development 131, 839-849 (2004).

26. W. Deng, H. F. Lin, miwi, a murine homolog of piwi, encodes a cytoplasmic protein essential for spermatogenesis. Developmental cell 2, 819-830 (2002).

27. T. Watanabe et al., MITOPLD Is a Mitochondrial Protein Essential for Nuage Formation and piRNA Biogenesis in the Mouse Germline. Developmental cell 20, 364-375 (2011).

28. H. Y. Huang et al., piRNA-Associated Germline Nuage Formation and Spermatogenesis Require MitoPLD Profusogenic Mitochondrial-Surface Lipid Signaling. Developmental cell 20, 376-387 (2011).

29. K. Zheng et al., Mouse MOV10L1 associates with Piwi proteins and is an essential component of the Piwi-interacting RNA (piRNA) pathway. Proceedings of the National Academy of Sciences of the United States of America 107, 11841-11846 (2010).

30. R. J. A. Frost et al., MOV10L1 is necessary for protection of spermatocytes against retrotransposons by Piwi-interacting RNAs. Proceedings of the National Academy of Sciences of the United States of America 107, 11847-11852 (2010).

31. M. Flemr et al., A Retrotransposon-Driven Dicer Isoform Directs Endogenous Small Interfering RNA Production in Mouse Oocytes. Cell 155, 807-816 (2013).

32. T. Watanabe et al., Endogenous siRNAs from naturally formed dsRNAs regulate transcripts in mouse oocytes. Nature 453, 539-543 (2008).

33. O. H. Tam et al., Pseudogene-derived small interfering RNAs regulate gene expression in mouse oocytes. Nature 453, 534-538 (2008).

34. M. Hirose, A. Ogura, The golden (Syrian) hamster as a model for the study of reproductive biology: Past, present, and future. Reprod Med Biol 18, 34-39 (2019).

35. K. Ishino et al., Hamster PIWI proteins bind to piRNAs with stage-specific size variations during oocyte maturation. Nucleic acids research, (2021).

36. Z. Q. Fan et al., Efficient Gene Targeting in Golden Syrian Hamsters by the CRISPR/Cas9 System. Plos One 9, (2014).

37. M. Hirose et al., Acrosin is essential for sperm penetration through the zona pellucida in hamsters. Proceedings of the National Academy of Sciences of the United States of America 117, 2513-2518 (2020).

38. B. Gu, E. Posfai, J. Rossant, Efficient generation of targeted large insertions by microinjection into two-cell-stage mouse embryos. Nat Biotechnol 36, 632-637 (2018).

39. N. Hustedt, D. Durocher, The control of DNA repair by the cell cycle. Nature cell biology 19, 1-9 (2016). 
40. J. Zenker et al., A microtubule-organizing center directing intracellular transport in the early mouse embryo. Science 357, 925-+ (2017).

41. D. Kigami, N. Minami, H. Takayama, H. Imai, MuERV-L is one of the earliest transcribed genes in mouse one-cell embryos. Biol Reprod 68, 651-654 (2003).

42. E. J. Grow et al., Intrinsic retroviral reactivation in human preimplantation embryos and pluripotent cells. Nature 522, 221-+ (2015).

43. A. A. Aravin et al., A piRNA pathway primed by individual transposons is linked to de novo DNA methylation in mice. Molecular cell 31, 785-799 (2008).

44. J. Brennecke et al., Discrete small RNA-generating loci as master regulators of transposon activity in Drosophila. Cell 128, 1089-1103 (2007).

45. K. N. Schulz, M. M. Harrison, Mechanisms regulating zygotic genome activation. Nature Reviews Genetics 20, 221-234 (2019).

46. E.-Z. Shen et al., Identification of piRNA Binding Sites Reveals the Argonaute Regulatory Landscape of the C. elegans Germline. Cell 172, 937-951.e918 (2018).

47. E. Taborska et al., Restricted and non-essential redundancy of RNAi and piRNA pathways in mouse oocytes. PLoS genetics 15, (2019).

48. Z. Loubalova et al., Golden hamster piRNAs are necessary for early embryonic development and establishment of spermatogonia. bioRxiv, 2021.2001.2027.428513 (2021).

49. H. Hasuwa et al., Production of functional oocytes requires maternally expressed PIWI genes and piRNAs in golden hamsters. bioRxiv, 2021.2001.2027.428354 (2021).

50. X. Z. G. Li et al., An Ancient Transcription Factor Initiates the Burst of piRNA Production during Early Meiosis in Mouse Testes. Molecular cell 50, 67-81 (2013). 


\section{Figure legend}

\section{Fig. 1 Generation of PIWIL1 mutant golden hamsters}

(A) Expression of PIWIs in mouse, golden hamster, and human MII oocytes. Expression levels are normalized by transcripts per million (TPM). (B) Composition of small RNA categories according to their size distributions in mouse, golden hamster, and human MII oocytes. (C) Strategy for the generation of PIWIL1 mutant golden hamsters by two-cell embryo CRISPR/Cas9 injection. The two-cell embryos from WT golden hamsters were collected and injected with Cas9 mRNAs and sgRNAs, which were then transferred into albino true-pregnant recipient females mated with albino males to obtain PIWIL1-edited founder pups. (D) Structure of the golden hamster PIWIL1 gene and generation of PIWIL1 mutants. Two PIWIL1 mutant strains were generated. Mutant1 (PIWIL1 $\left.{ }^{\mathrm{m} 1 / \mathrm{m} 1}\right)$ contained a $7 \mathrm{nt}$ deletion and a $15 \mathrm{nt}$ insertion in exon 17. Mutant2 (PIWIL $\left.1^{\mathrm{m} 2 / \mathrm{m} 2}\right)$ contained an 11 nt deletion in exon 6. Both of the mutations caused a frameshift in the PIWIL1 mRNA resulting in a premature stop codon. (E) Western blots showing the loss of PIWIL1 expression in ovary, MII oocyte, one-cell embryo, two-cell embryo, and testis of PIWIL $1^{\mathrm{m} 1 / \mathrm{m} 1}$ hamsters. ACTB was used as the loading control. (F-G) Immunostaining shows loss of PIWIL1 expression in the mutant ovary (F) and testis (G). Scale bars $=100 \mu \mathrm{m}$ (top); Scale bars $=25 \mu \mathrm{m}$ (bottom).

\section{Fig. 2 PIWIL1 deficiency causes infertile in both male and female golden hamsters}

(A) Fecundity of male and female PIWIL1 mutant golden hamsters. Error bars indicate standard error of mean (s.e.m). (B) Embryogenesis ratio of PIWIL1 $1^{\mathrm{m} 1 / \mathrm{m} 1}$ embryos transferred to WT recipients. $\mathrm{n}$ is the number of embryos at each indicated stage. (C) The development of PIWIL1 mutant embryos was arrested at the two-cell stage in vivo. MII oocytes and embryos from the oviducts of WT and PIWIL $1^{\mathrm{m} 1 / \mathrm{m} 1}$ females mated with WT males were collected at $0,9,33,44$, and 52 hours post egg activation (PEA). Scale bar $=50 \mu \mathrm{m}$. (D) Embryonic development of PIWIL1 mutants was arrested at the two-cell stage in vivo and in vitro. Embryos from WT or PIWIL $1^{\mathrm{m} 1 / \mathrm{m} 1}$ females mated with WT males were collected at 33 or 52 PEA and their in vivo embryogenesis ratio was calculated. For in vitro analysis, zygotes from WT and PIWIL $1^{\mathrm{m} 1 / \mathrm{m} 1}$ females mated with WT males were collected at 9 PEA and cultured in vitro; embryogenesis ratios were calculated at 33 and 52 PEA. (E) The ratio of pups resulting from WT embryos transferred to WT and PIWIL $1^{\mathrm{m} 1 / \mathrm{m} 1}$ 
recipients, respectively.

\section{Fig. 3 PIWIL1-piRNAs are indispensable for suppression of ERVs but not for LINE1s}

(A) Composition of small RNA categories according to their length distribution. Early-stage oocytes were collected from the ovaries, and MII oocytes were collected by superovulation. The embryos were collected from the oviducts of WT and PIWIL $1^{\mathrm{m} 1 / \mathrm{m} 1}$ females mated with WT males. The average of 2-6 biological replicates are shown. The small RNA counts were normalized by exogenous spike-in. PF, primary follicle stage oocyte; SF, secondary follicle stage oocyte; GV, germinal vesicle stage oocyte; MII, metaphase II oocyte; $1 \mathrm{C}$, one-cell embryo; $2 \mathrm{C}$, two-cell embryo; 4C, four-cell embryo. (B) Differential analysis of consensus TE expression in WT and PIWIL $1^{\mathrm{m} 1 / \mathrm{m} 1}$ oocytes and embryos. The expression of TEs was normalized by exogenous spike-in. The highly significant up- or down-regulated TEs ( $\geq 2$ folds; -value $<0.05$ determined by Welch two sample $t$-test) are indicated in red or blue, respectively, and the TE number is shown at the top. (C) The bar graphs show the expression level (RPKM) of piRNAs mapped to the sense (gray) and antisense (red) direction of each TE family. PIWIL1 29 nt-piRNAs, PIWIL1 23 nt-piRNAs, and PIWIL3 19 ntpiRNAs derived from different TE families are plotted. The significantly up-regulated TE families are listed with fold changes of expression level in PIWIL $1^{\mathrm{m} 1 / \mathrm{m} 1}$ versus WT MII oocytes. The $\log 2$ fold change level is indicated as the degree of orange in the heatmap. (D) The Ping-Pong signature of PIWIL1-piRNAs (23 nt-piRNA and 29 nt-piRNA) and PIWIL3-piRNAs (19 nt-piRNA) derived from up-regulated LTR/ERV and LINE1 subfamily members in WT and PIWIL1 $1^{\mathrm{m} 1 / \mathrm{m} 1}$ MII oocytes. The level of Ping-Pong signature is represented by the $Z$-score of 10 -nt overlapped piRNAs from opposite strands, with piRNAs having overlaps of different lengths serving as the background. Zscore $>1.96$ corresponds to $\mathrm{p}$-value $<0.05(50)$.

\section{Fig. 4 PIWIL1-piRNAs participate in the degradation of maternal mRNAs}

(A) PCA analysis of all genes expressed in the oocytes and embryos at different stages. The circles represent WT, and triangles represent PIWIL1 mutants. The gene expression levels were normalized by exogenous spike-in. (B) Pseudotime analysis of all gene expression by Monocle reveals a bifurcation between the developmental trajectories of WT and PIWIL1 $1^{\mathrm{m} 1 / \mathrm{m} 1}$ embryos into two 
distinct branches after 9 PEA. (C) Volcano plots show the differentially expressed genes in oocytes or embryos between GV and MII stages, MII stage and 9 PEA, 9 PEA and 33 PEA, 33 PEA and 44 PEA. The highly significant up- or down-regulated genes ( $\geq 2$ folds; Welch two sample $t$-test, pvalue $<0.01)$ are indicated in red or blue, respectively, with gene number shown at the top. (D) Degradation of some maternal mRNAs is inhibited between the MII stage to 9 PEA in PIWILI mutants. X- and Y-axes represent the $\log 2$ fold change of gene expression in WT (X-axis) and PIWILI $1^{\mathrm{m} 1 / \mathrm{m} 1}$ (Y-axis) at 9 PEA versus MII. (E) The number of targets on the mRNAs matched to significantly down-regulated piRNAs after PIWIL1 knockout was correlated with the level of mRNA up-regulation. The level of target mRNA up-regulation was calculated as the ratio of fold changes in embryos at 9 PEA to MII oocytes between PIWIL $1^{\mathrm{m} 1 / \mathrm{m} 1}$ and WT samples. 


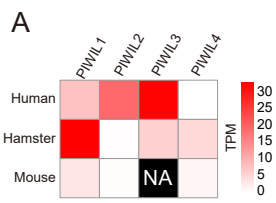

B

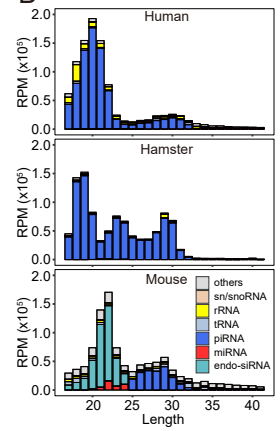

$\mathrm{F}$

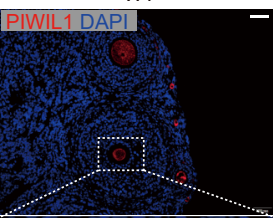

C

Day 1: supervolation

$$
\text { Day 5: mating }
$$

D

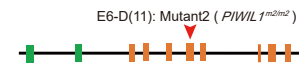
$\left.n^{2}\right)$

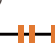

Target sequence

PAM

WT: AGCTGGCGATGATTGTGGGCATCGACTGTTACCATGACACCACAGCTGGGCGGAGGT

\section{$\downarrow$}

Mutant1: AGCTGGCGATGATTGTGGGCATCGACTGTTACCATGACACCACGATACGACT-GCAGGTCGGAGGT

E
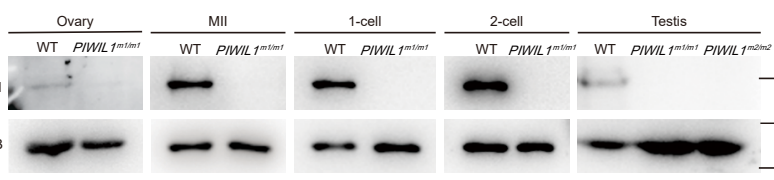

PIWIL1

ACTB

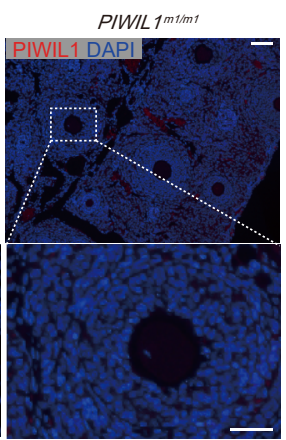

G

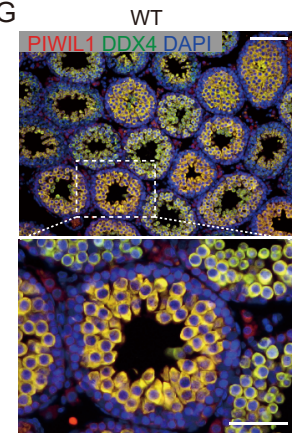

Golden hamster Albino golden hamster

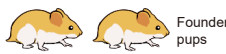
P.

E17-INS(15)D(7): Mutant1 ( $P / W / L 1^{\text {mlims }}$ ) $\checkmark$

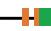

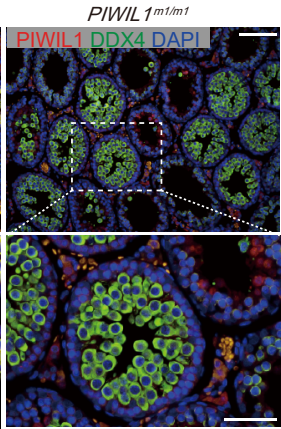

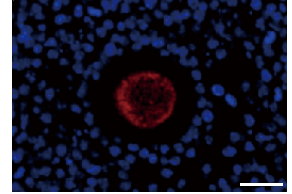


A
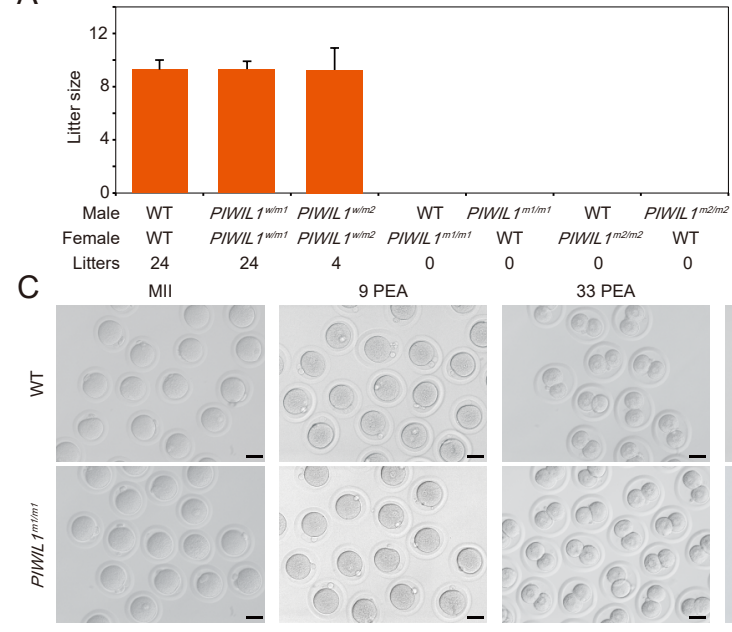

D

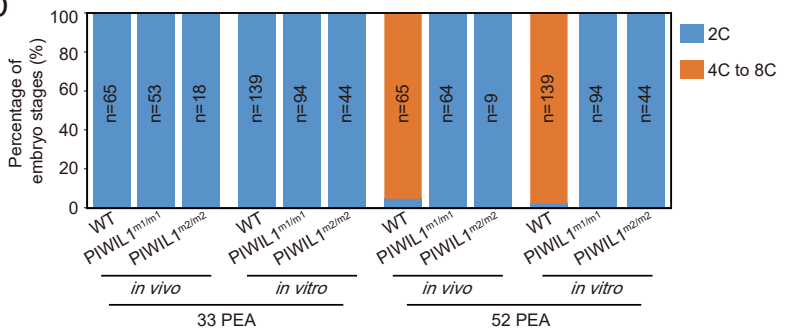

B

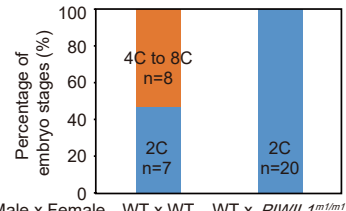

Male $x$ Female WT $\times$ WT WT $\times$ PIWIL $1^{\text {mt/mt }}$

$\begin{array}{rrr}\text { No. embryos } & 15 & 20 \\ \text { Recipient } & \text { WT } & \text { WT }\end{array}$

44 PEA

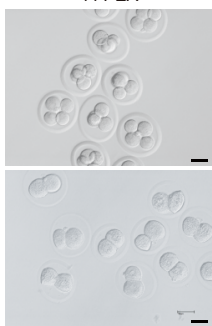

E

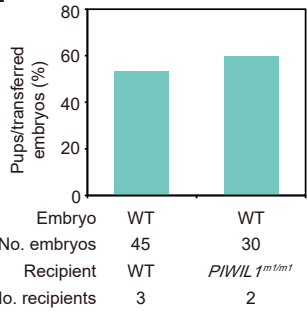



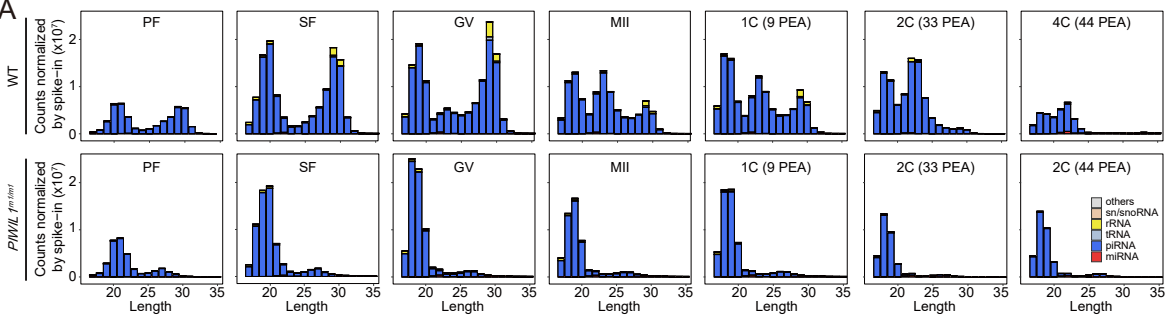

B
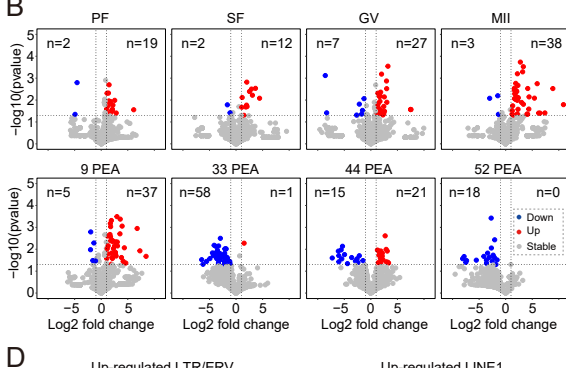

$\mathrm{D}$

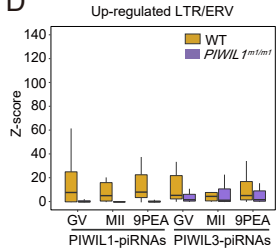

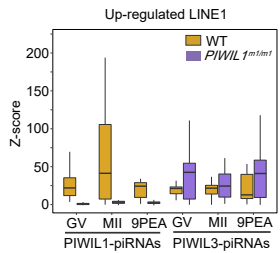
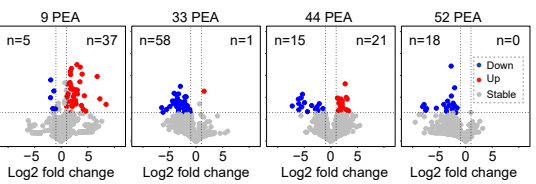

C
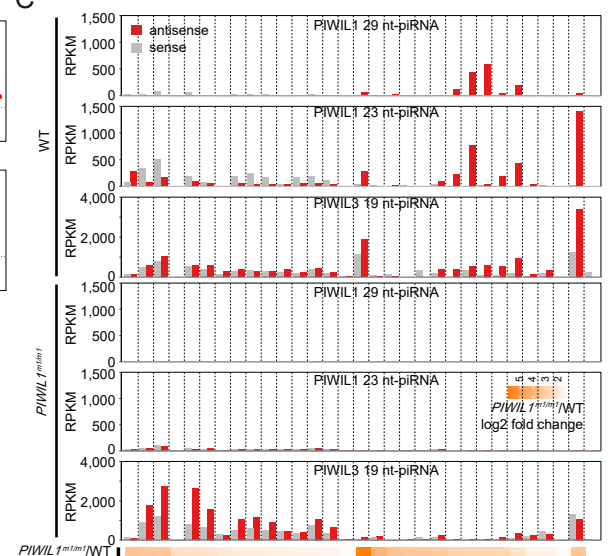

PIWIL $1^{m t / m} /$ WT
$\log 2$ fold change

LINE1

LTR/ERV

LTR/others 


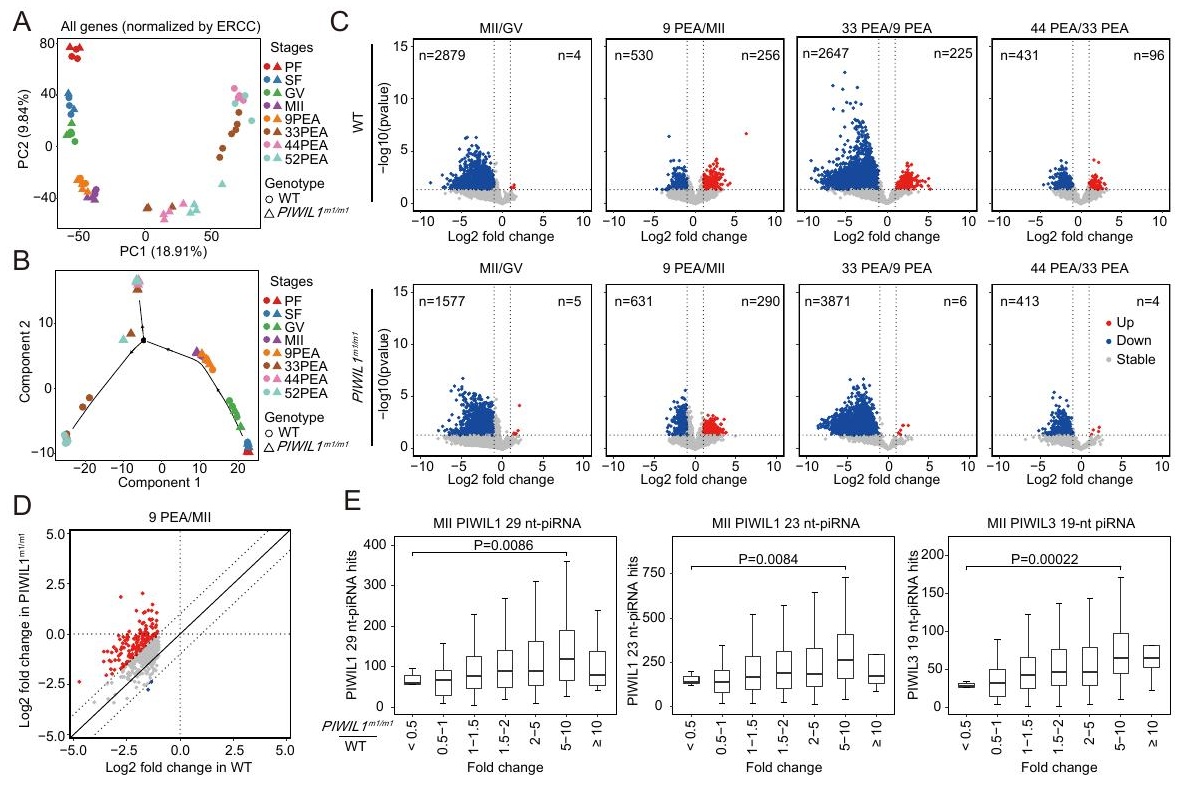

